\title{
Solutions Space State Analysis for Transmission Minimizing of Quasi One-Dimensional Phononic Structures
}

\author{
S. GARUS \\ Department of Mechanics and Fundamentals of Machinery Design, \\ Czestochowa University of Technology, Poland \\ Doi: $10.12693 /$ APhysPolA.138.299 \\ *e-mail: gari.sg@gmail.com
}

\begin{abstract}
In the paper, all possible transmissions of quasi one-dimensional structures permutations made of glass and epoxy resin surrounded by water for the frequency range of acoustic waves was determined using the Transfer Matrix Method (TMM) algorithm. On their basis, the phase space of solutions for minimizing the objective function for the genetic algorithm was determined.This space consisted of the transmission integral (energy reduction of propagating wave) and the integral of the absolute value of the transmission functions derivative (elimination of high transmission peaks with a small half width). The paper demonstrates the existence of bandgaps for the analyzed multilayer structures (waves with characteristic frequencies do not propagate in the structure) and demonstrates the existence of local minima of solution spaces with low probability of finding optimal structure by the genetic algorithm. State space search for various initial values of the optimization algorithm was also analyzed.
\end{abstract}

topics: transmission, transfer matrix, genetic algorithm, multilayers, phononic structures

\section{Introduction}

Phononic structures are composites in which mechanical waves of given frequencies do not propagate, this phenomenon is called the occurrence of phononic bandgap (PhBG) [1]. Phononic structures are used as acoustic waveguides, acoustic/elastic filters, noise control or can be used for demultiplexing [1-4]. How a mechanical wave propagates in a phononic crystal depends on the properties of the materials used but it is most influenced by the spatial distribution of the materials $[4,5]$. Depending on the structure of the phononic crystals, they can be divided into quasi one-dimensional (multilayer systems), two-dimensional or three-dimensional [4]. In many works the influence of layer distribution on the phononic properties of the analyzed structures has been shown $[6,7]$. In order to obtain the desired structure properties, optimization algorithms with a properly selected objective function are used [8].

\section{Mathematical models}

The work analyzes the distribution in the phase space the constituents of the objective function so that the mechanical wave transmission in a given frequency area is minimized, as well as the elimination of narrow high transmission bands. The genetic algorithm (GA) was used as the optimization algorithm, and the transmission of the analyzed structures in the frequency domain was determined by the Transfer Matrix Method (TMM) algorithm. The influence of various initial conditions on searching the space of solution states was also analyzed.

\subsection{Transfer Matrix Method}

Mechanical wave propagation in phononic structures can be described using a matrix equation

$$
\left[\begin{array}{c}
p_{\text {in }}^{+} \\
p_{\text {in }}^{-}
\end{array}\right] M=\left[\begin{array}{c}
p_{\text {out }}^{+} \\
0
\end{array}\right]
$$

where $p_{\text {in }}^{+}$is the amplitude of the wave incident on the structure $p_{\text {in }}^{-}$is the amplitude of the reflected wave, and $p_{\text {out }}^{+}$is the amplitude of the wave propagating behind the structure. The $M$ matrix depends on the properties of the materials forming the structure and their distribution. It consists of wave propagation matrix $\Gamma_{i}$ inside layer $i$ and transmission matrix $\Phi_{i, i+1}$ between layers $i$ and $i+1$. The in and out are the materials surrounding the multilayer structure. The characteristic matrix consisting of in layers was determined by

$$
M=\Phi_{\mathrm{in}, 1}\left[\prod_{i=1}^{n-1} \Gamma_{i} \Phi_{i, i+1}\right] \Gamma_{n} \Phi_{n, \text { out }}
$$

Transmission for a given frequency $f$ is determined from the first word of the characteristic matrix main diagonal $M_{11}$

$$
T_{f}=\left|\frac{1}{M_{11}}\right|^{2} .
$$

The propagation matrix $\Gamma_{i}$ is determined by the frequency $f$, phase velocity of mechanical wave $v_{i}$, the thickness of a given layer $d_{i}$ and takes the form

$$
\Gamma_{i}=\left[\begin{array}{cc}
\mathrm{e}^{\mathrm{i} 2 \pi f \frac{d_{i}}{v_{i}}} & 0 \\
0 & \mathrm{e}^{-\mathrm{i} 2 \pi f \frac{d_{i}}{v_{i}}}
\end{array}\right]
$$



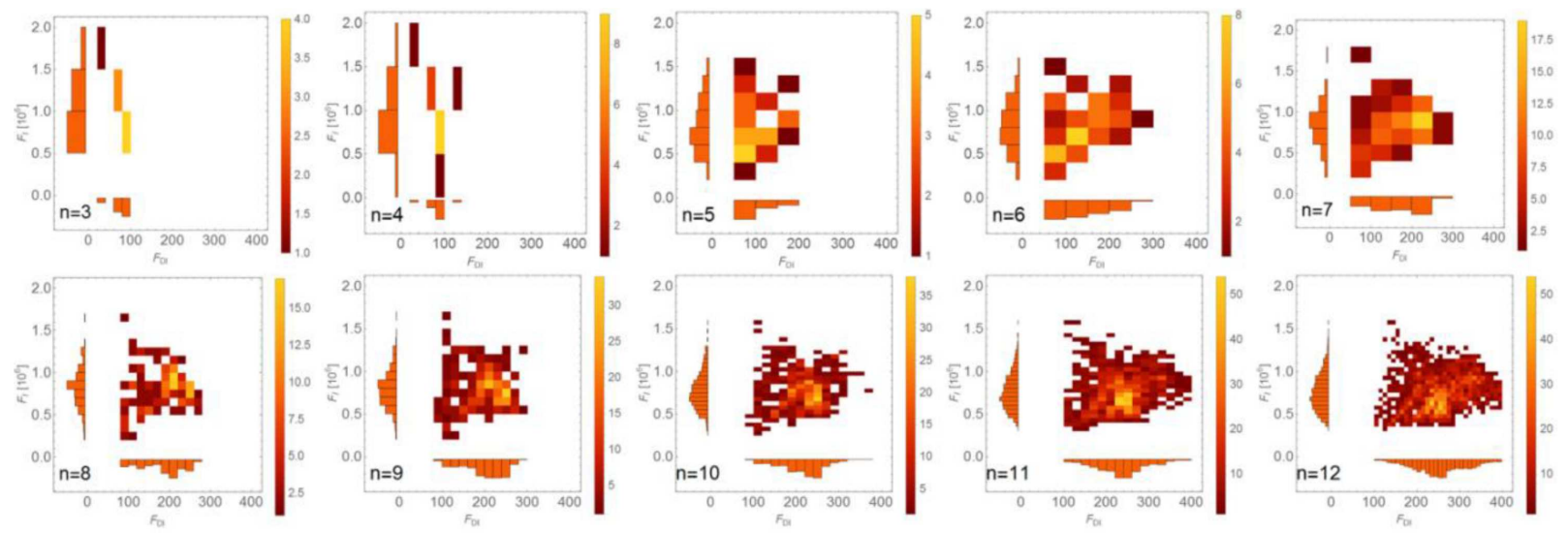

Fig. 1. Histograms of the objective function component distribution for different amounts of layers $n$ in the structure.

The transmission matrix $\Phi_{i, i+1}$ between layers $i$ and $i+1$ is given by

$$
\Phi_{i, i+1}=\frac{1}{t_{i, i+1}}\left[\begin{array}{cc}
1 & r_{i, i+1} \\
r_{i, i+1} & 1
\end{array}\right]
$$

where

$$
r_{i, i+1}=\frac{Z_{i+1}-Z_{i}}{Z_{i+1}+Z_{i}}
$$

and

$$
t_{i, i+1}=\frac{2 Z_{i+1}}{Z_{i+1}+Z_{i}}
$$

The $t_{i, i+1}$ and $r_{i, i+1}$ are the transmission and reflectance coefficients at the boundaries of layers $i$ and $i+1$, respectively. The acoustic impedance $Z_{i}$ for layer $i$ is determined by the medium phase velocity $v_{i}$ and its mass density $\rho_{i}$, and it is defined as $Z_{i}=v_{i} \rho_{i}$.

\subsection{Genetic algorithm}

The genetic algorithm is used to design the distribution of layers in a quasi-one-dimensional structure so that the desired mechanical wave propagation properties are obtained. This algorithm works as follows:

\section{Initialization.}

2. The random selection of the first population of genotypes for analysis.

3. Determination of transmission for table of structures using TMM.

4. Determining the components of the objective function and calculating its value,

5. Sorting the genotype table by objective function.

6. Leaving the two most favorable structures unchanged, and mixing the remaining genotypes, where the probability of mixing depends on the quality of the structures.

7. Gene mutation of the entire population with a given probability.

8. If the limit of loop runs has been reached, the analysis is terminated, otherwise return to point III.
The selection of the objective function that determines the algorithm's course is extremely important. The objective functions $F_{C}$ used consists of the product of two normalized functions $F_{I}$ and $F_{D I}$. The normalization process affects the equal participation of both functions in the objective function, namely

$$
F_{C}=\left\|F_{I}\right\|\left\|F_{D I}\right\| \text {. }
$$

This is beneficial for the algorithm in comparing the quality of functions within one population and during gene mixing. However, comparing structure quality across populations should be done without normalizing according to

$$
F_{C}^{\prime}=F_{I} F_{D I},
$$

where functions $F_{I}$ and $F_{D I}$ are used to analyze the space of solutions. They are defined as

$$
\begin{aligned}
& F_{I}=\int_{f_{\min }}^{f_{\max }} T(f) \mathrm{d} f \\
& F_{D I}=\int_{f_{\min }}^{f_{\max }}\left|\frac{\partial T(f)}{\partial f}\right| \mathrm{d} f
\end{aligned}
$$

Thus, $F_{I}$ is responsible for minimizing transmission in a specific frequency range, while $F_{D I}$ allows the elimination of high transmission narrow peaks.

\section{Research}

The analysis was carried out for the frequency range up to $20 \mathrm{kHz}$. In simulation, glass was adopted as material A (with $v_{A}=4000 \mathrm{~m} / \mathrm{s}$, and $\left.\rho_{A}=3880 \mathrm{~kg} / \mathrm{m}^{3}\right)$, while epoxy resins as material B (with $v_{B}=2535 \mathrm{~m} / \mathrm{s}$ and $\rho_{B}=1180 \mathrm{~kg} / \mathrm{m}^{3}$ ). The structure was surrounded by water $\left(v_{w}=\right.$ $\left.1500 \mathrm{~m} / \mathrm{s}, \rho_{w}=1000 \mathrm{~kg} / \mathrm{m}^{3}\right)[9,10]$. The thickness of the layers was $1 \mathrm{~cm}$. The analysis was carried out for the number of layers $n$ in the range from 3 to 12. All possible distributions of $\mathrm{A}$ and $\mathrm{B}$ materials were determined and the transmission of acoustic waves was calculated for them. The values of $F_{I}$ 

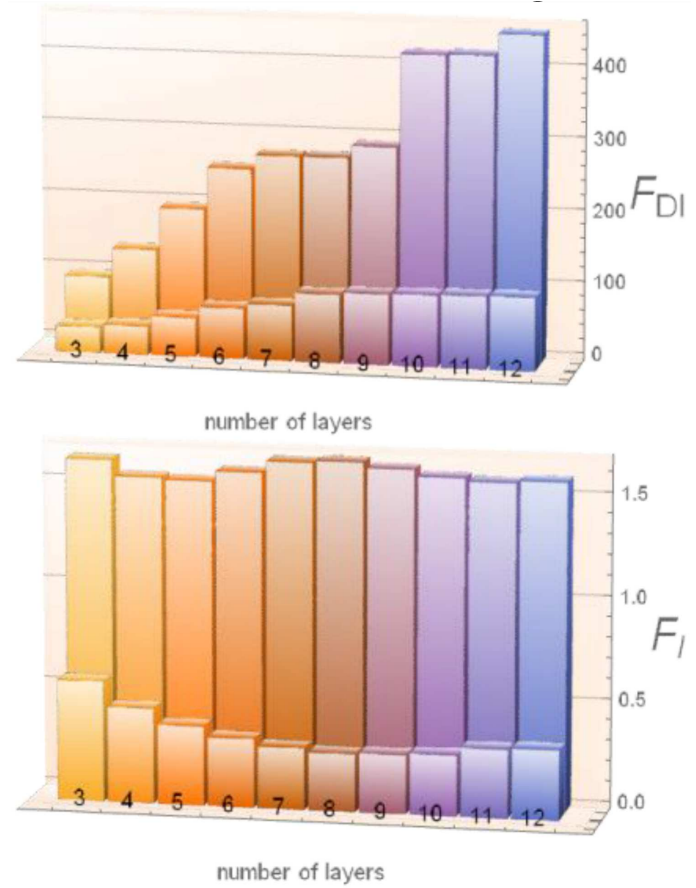

Fig. 2. Impact of the number of layers on the maximum and minimum values of the objective function components.

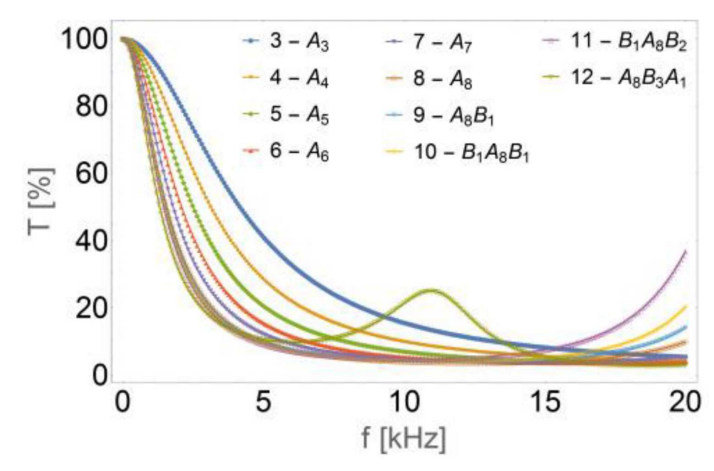

Fig. 3. Transmission of structures with the smallest $F_{I}$ for subsequent numbers of layers $n$ from 3 to 12 .

and $F_{D I}$ parameters were determined and collected on the histogram diagrams of the phase space solutions (Fig. 1). The minimum and maximum values of the components of the objective function are collected in Fig. 2.

Figure 3 presents transmission charts for structures with the lowest value of the objective function for a given number of layers. The subscript in the structure record corresponds to the number of times the layer is repeated. Then, using GA, space search of solutions for 20 individuals in the population with $1 \%$ mutation chance was introduced (Fig. 4). At each step, the 2 best structures were left, and the 2 weakest were replaced by random ones. The 2.2 percent of the state space was searched.
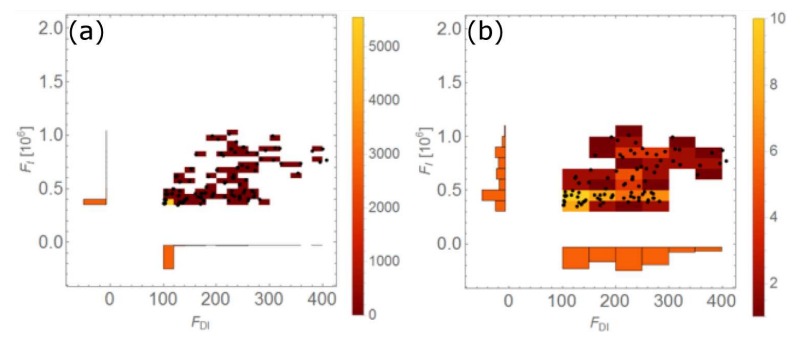

Fig. 4. Histograms of the objective function values for structures analyzed during the operation of the genetic algorithm for (a) all analyzed layer distributions and (b) unique structures.

\section{Conclusions}

Increasing the number of layers in the structure resulted in the occurrence of more narrow peaks with high transmission (increase in $F_{D I}$ ). The distribution of the components of the objective function did not correspond to the Gaussian distribution. For the number of layers from 6 to 11 there was a band gap in the range from $10 \mathrm{kHz}$ to $15 \mathrm{kHz}$, while for 12 layers a broad peak was observed for the frequency of $11 \mathrm{kHz}$ and the transmission level of $25 \%$. Based on the analysis of the behaviour of the genetic algorithm, it would be advisable to increase the number of random structures added to the population. This would ensure greater coverage of state spaces and avoid local minima of solution spaces.

\section{References}

[1] I. Kriegel, F. Scotognella, Physica E 85, 34 (2017).

[2] S. Villa-Arango, R. Torres, P.A. Kyriacou, R. Lucklum, Measurement 102, 20 (2017).

[3] A. Cicek, O.A. Kaya, M. Yilmaz, B. Ulug, J. Appl. Phys. 111, 013522 (2012).

[4] M. Tianxue, S. Xiaoxing, D. Haowen, W. Yuesheng, Z. Chuanzeng, Chinese J. Theor. Appl. Mech. 49, 743 (2017).

[5] Z. Zhang, Y. F. Li, F. Meng, X. Huang, Comput. Mater. Sci. 139, 97 (2017).

[6] S. I. Fomenko, M.V. Golub, A. Chen, Y. Wang, C. Zhang, J. Sound Vib. 439, 219 (2019).

[7] I. Kriegel, F. Scotognella, Physica E 85, 34 (2017).

[8] S. Hedayatrasa, K. Abhary, M. Uddin, Ultrasonics 57, 104 (2015).

[9] S. Garus, W. Sochacki, M. Bold, Eng. Mech. 2018, 229 (2018).

[10] S. Garus, W. Sochacki, J. Appl. Math. Comput. Mech. 17, 19 (2018). 